\title{
A formação do conceito de ontologia na ciência da informação: uma análise nos periódicos Scire e Ibersid
}

\author{
La formación del concepto de ontología en la ciencia de la información: un análisis de las revistas Scire e Ibersid
}

The formation of the concept of ontologies in the information science: an analysis on the journals Scire and Ibersid

\author{
Walter MOREIRA (1), Martins Fideles dos SANTOS Neto (2)
}

\begin{abstract}
Departamento de Ciência da Informação, Faculdade de Filosofia e Ciências, Universidade Estadual
Paulista (Unesp), Marília, Brasil; (1) walter.moreira@marilia.unesp.br; (2) martins_neto17@hotmail.com
\end{abstract}

\begin{abstract}
Resumen
Se analiza el concepto, todavia inestable, de ontología como instrumento de organización y representación del conocimiento en dos revistas españolas del área de documentación, a saber, las colecciones completas de "Scire: representación y organización del conocimiento " e "lbersid: revista de sistemas de información y documentación", publicadas por la Universidad Zaragoza. Se buscó el término "ontología" en los campos de título, palabras-clave y resumen, obteniéndose un corpus de dieciocho artículos disponibles en formato electrónico. El análisis temático del corpus reveló que lo enfoques sobre ontologías se distribuyeron en cuatro categorías de análisis: conceptualización, comparación, aplicación y nuevas aplicaciones. Se observó, además, que la manera como se manejan las cuestiones acerca de las ontologías en las dos revistas se relaciona con la política editorial que desarrollan. Los resultados permitieron concluuir que existe un proceso de metaterminologización del término ontología, desde la filosofía hacia a la documentación, que merece estudios adicionales más verticales.
\end{abstract}

Palabras clave: Ontologías. Organización del conocimiento. Representación del conocimiento. Scire. Ibersid.

\section{Introdução}

Um campo de conhecimento pode ser compreendido, dentre outros modos possíveis, por meio da sua epistemologia, identificando-se as teorias que lhe sustentam e as novas propostas teóricas que orientam seu desenvolvimento. É possível também compreendê-lo por meio da sua metodologia, tomando-se essa palavra em sua acepção mais próxima do seu sentido original, isto é, de estudo dos métodos empregados na identificação, na descrição e na solução dos problemas que caracterizam seu objeto de estudo.

Desse modo, e na linha que propõe Gnoli (2011), o campo da organização do conheci-

\begin{abstract}
The concept of ontoloy as a tool for knowledge organization and representation, which is not yet stable, is analyzed in two spanish journals of information science, namely "Scire: representación y organización del conocimiento" and "Ibersid: revista de sistemas de información y documentación", both published by the University of Zaragoza. The term "ontology" and its variations were searched in the fields of title, keywords and abstract. A corpus of eighteen articles was obtained. The approaches to ontologies were classified into four categories of analysis: conceptualization, comparison, application and new applications. It was observed that the way which the concept of ontologies is treated in both periodicals is in consonance with their editorial policies. The term ontology is suffering a process of meta-terminologization, from the field of philosophy towards information science, a fact that deserves further and more vertical studies.
\end{abstract}

Keywords: Ontologies. Knowledge organization. Knowledge representation. Scire. Ibersid.

mento, deve ser compreendido em seus aspectos ontológico (que envolve a compreensão do fenômeno estudado), epistemológico (a perspectiva sob a qual o fenômeno é estudado) e pragmático (envolvendo usuários e aspectos materiais dos documentos).

A organização do conhecimento pode também ser compreendida em sentido amplo e em sentido estrito, conforme a proposta de Hjorland (2008). Em sentido amplo, diz respeito à organização social do conhecimento, ao modo como o conhecimento é estruturado com o fim de atender às identificações de disciplinas e profissões. Em sentido estrito a organização do conhecimento compreende atividades como (Hjorland, 2008, p. 86, tradução livre): 
[...] descrição documental, indexação e classificação executadas em bibliotecas, bases de dados bibliográficos, arquivos e outros tipos de 'instituições de memória', por bibliotecários, arquivistas, especialistas em informação, especialistas em determinados assunto, bem como por algoritmos computacionais e leigos.

A discussão que o presente trabalho estabelece envolve o conceito de ontologia e se refere mais proximamente, portanto, ao aspecto estrito da organização do conhecimento, pois considerase para os fins dessa discussão que as ontologias são instrumentos de organização e representação do conhecimento.

Observando-se, portanto, o aspecto representacional presente na concepção de Hjorland (2008), o qual pode ser destacado na expressão "descrição documental, indexação e classificação", opta-se, nesse trabalho, pelo termo "organização e representação do conhecimento" (ORC) para se referir à organização do conhecimento em sentido estrito. Compreendida nesse sentido, a organização do conhecimento encontra na ciência da informação a disciplina com a qual mantém as principais relações de interface.

$\mathrm{Na}$ acepção mais literal de Kiel (1994), organizar o conhecimento significa tornar o conhecimento um órgano (do grego organon, cujo sentido remete ao de instrumento), visando a atender determinados interesses identificados.

Nesse sentido, considerando-se a aproximação pragmática imediata que se faz entre a organização do conhecimento e seus instrumentos, colocam-se duas questões como vetores: quais os instrumentos utilizados nos processos de organização e representação do conhecimento e qual a eficácia desses instrumentos em seus aspectos ontológico, epistemológico e pragmático? Tendo-se em vista que o mapeamento a que remete a primeira questão já está realizado de forma relativamente suficiente nos diversos estudos sobre a temática e que a segunda questão não pode ser alcançada do modo amplo como está em estudos isolados, propõe-se nesse trabalho uma terceira questão que contribui teoricamente para o alcance desta última. Tomando-se as ontologias como instrumentos de organização e representação do conhecimento e considerando-se que a formação do conceito de ontologia, sendo observado de modo focalizado, ocorre no discurso da ciência da informação, propõe-se como problema geral a identificação do conceito de ontologia que o campo veicula. Como elementos de materialização discursiva e corpus documental, são considerados dois periódicos especializados.
Propôs-se como objetivo, portanto, verificar a configuração do conceito de ontologia em dois periódicos espanhóis que publicam, entre outras contribuições, os trabalhos selecionados para apresentação no Ibersid, respectivamente as revistas Scire: representación y organización del conocimiento e lbersid: revista de sistemas de información y documentación, ambos publicados pela Universidade de Zaragoza, com periodicidade, respectivamente, semestral e anual. Ambas preocupadas com as questões que envolvem a ciência da informação, a primeira de alcance mais especializado e a segunda de alcance mais amplo, como denunciam os subtítulos.

Para a consecução dos objetivos, utilizou-se a metodologia de análise de conteúdo (Bardin, 2011), buscando-se identificar a abordagem sobre ontologias nas seguintes categorias: conceitualização, comparação, aplicação e nova aplicação. As descrições sobre essas categorias são dadas na seção 4 desse trabalho.

A análise contemplou a totalidade dos artigos disponíveis em formato eletrônico, considerando-se o período 1995-2013 para a Scire e o período 2007-2013 para a Ibersid. O corpus documental total foi composto de 565 artigos (incluindo investigações em curso, artigos breves e comunicações), distribuídos da seguinte forma: 365 artigos da Scire e 200 artigos da Ibersid.

Utilizou-se como estratégia de busca a identificação dos termos "ontologia", "ontología" e "ontology", com suas variações de número, nos campos de título, resumo e palavras-chave. As buscas foram realizadas com recurso da ferramenta de busca disponível nas páginas dos periódicos em análise. Inicialmente, utilizandose apenas o critério de ocorrência do termo, foram identificados 42 artigos (26 localizados na Scire e 16 localizados na Ibersid). Após leitura seletiva e análise desse material, foram identificados os artigos que apresentavam ou desenvolviam de algum modo o conceito de ontologia, conforme os interesses dessa pesquisa. Selecionou-se, desse modo, o corpus de análise, composto de dezoito artigos (doze localizados na Scire e seis localizados na Ibersid). Os dezoito artigos foram analisados com o fim de verificar e categorizar a abordagem que o termo "ontologia" recebia no texto.

Uma vez que se estabelece uma discussão conceitual sobre as ontologias, considera-se necessário discorrer sobre o lugar das ontologias na ciência da informação, em sua condição de instrumento (objeto da seção 2) e sobre a importância da análise do conceito em si (objeto 
da seção 3) antes da apresentação dos resultados (objeto da seção 4).

\section{A ontologia no escopo da organização do conhecimento}

A ontologia, analisada em seu sentido filosófico, tem origem na Grécia Antiga e centra sua preocupação na natureza e na realidade dos seres. Após as contribuições de diversos pensadores, dentre os quais Aristóteles, Descartes, Kant, Brentano, Husserl, além dos escolásticos da Idade Média (Smith, 2003), é perfeitamente compreensível e até mesmo esperado que o conceito contemporâneo de ontologia, na condição mesma de conceito filosófico, não seja pacífico. Tal conceito, entretanto, está fundado numa vasta, rica e longeva literatura sobre a questão, o que, se não elucida a questão, certamente auxilia no seu esclarecimento.

A literatura sobre ontologias na ciência da informação ainda é relativamente incipiente. Esse fato pode ser encarado como um processo natural de maturação, pois as discussões em torno da temática ainda são recentes e o conceito ainda se encontra em fase de maturação.

$\mathrm{Na}$ interface teórico-conceitual da ciência da informação com a ciência da computação, as ontologias são consideradas como artefatos desenhados especificamente para possibilitar a representação computacional de algum fenômeno. As ontologias são, portanto, modelos de algum aspecto da realidade, nos quais os conceitos são definidos de modo lógico-semântico em termos de seus limites e de suas correlações.

Embora sejam desenvolvidas em linguagens de programação e estruturadas para o compartiIhamento de informações entre máquinas, as ontologias computacionais não podem prescindir de um nível ontológico básico (no sentido filosófico), pois dependem do compartilhamento consensual de conceitos entre pessoas, ou seja, são devedoras de um "compromisso ontológico" (Quine, 1975), que possa dar garantias de intelecção, comunicação, compartilhamento e reuso.

As ontologias computacionais são utilizadas como instrumentos de construção de modelos a partir dos quais se pode organizar e representar o conhecimento. Se o processo de conhecer pode ser compreendido como um processo de construção de modelos de um dado domínio (Campos, 2004), pode-se afirmar que as ontologias também são instrumentos de cognição, a partir dos quais é possível compreender o mundo observável.
Um ponto comum entre ontologias e linguagens documentárias é que ambas são linguagens construídas para fins específicos. As ontologias, assim como as linguagens documentárias, na condição de instrumentos de representação do conhecimento, permitem descrever alguns fenômenos e atuam, desse modo, como linguagem.

Para Gardin, em cujos estudos o termo tem origem, uma linguagem documentária "é um conjunto de termos, providos ou não de regras sintáticas, utilizadas para representar conteúdos de documentos técnico-científicos com fins de representação ou busca retrospectiva de informações" (Lara, 2009, p. 63).

Enquanto as linguagens documentárias são utilizadas para construir representações que permitam traduzir o conteúdo dos documentos, ou seja, são aplicadas de forma prioritária em contextos documentários, as ontologias, numa classificação que privilegia seu nível de generalidade e seu propósito (Guarino, 1998; Staab; Studer, 2009), são utilizadas para descrever: a) conceitos genéricos, independentes de domínio, como, e. g., espaço, tempo e ação; b) conceitos especializados no interior de domínios, como o da medicina e.g.; c) conceitos especializados identificados em tarefas ou atividades, como, e. g., venda ou diagnóstico e d) conceitos que se relacionam tanto da identificação do domínio particular quanto da tarefa, os quais se referem, normalmente, a papéis desempenhados por atores do domínio. Essas ontologias são denominadas, respectivamente, ontologias de alto nível, ontologias de domínio, ontologias de tarefas e ontologias de aplicações.

O conceito de ontologia, no campo da ciência da informação, ainda não é estável e ainda se persegue sua identidade por meio de comparação com outros instrumentos de organização e representação do conhecimento. A instabilidade conceitual pode ser encarada de dois modos: ignorando-se-a e optando por esta ou aquela concepção ou, alternativa que desponta como mais adequada à postura científica, tomando-se a própria instabilidade como elemento de formação do conceito, em perspectiva dialética.

\section{A formação dos conceitos e sua importância para a formação do conhecimento científico}

A formulação e a análise do conceito (Semidão; Almeida; Moreira, 2013) atuam como elemento de ruptura epistemológica com o senso comum, ou seja, a precisão conceitual está dialeticamente relacionada à identificação precisa do objeto que caracteriza uma determinada ciência. Uma ciência, ensina Benveniste (1989, p. 252), "só 
começa a existir ou consegue se impor na medida em que faz existir e em que impõe seus conceitos". A formação e a estabilização dos conceitos científicos ocorrem por meio de processos de vocabularização (a passagem da terminologia para a língua comum), terminologização (a passagem da língua comum para a terminologia) e metaterminologização (a passagem de uma terminologia a outra) (Krieger; Finatto, 2004; Barbosa, 2005).

As negociações de sentido necessárias aos processos de comunicação científica demandam identificações precisas de conceitos. A precisão conceitual, nesse caso, deve ser entendida como instrumento para o diálogo, posto que o conceito científico está em permanente objetivação. De outro modo, cada cientista será sempre obrigado a "tentar construir seu campo de estudos começando pelos primeiros princípios e justificando o uso de cada conceito introduzido" (Kuhn, 1978, p. 40).

Entende-se que o termo "ontologia" sofre um caso clássico de metaterminologização e exatamente por isso requer cuidados referentes a sua significação e ao sentido que ganha no discurso científico da ciência da informação.

A presença de uma terminologia própria e de um sistema conceitual preciso, robusto e coerente são marcas características de um campo científico bem demarcado. A demarcação, nesse caso, não deve ser tomada como condição de isolamento, como fator de impedimento de importação e exportação de termos e conceitos. Ao contrário, apenas a partir do conhecimento preciso da própria terminologia (postura disciplinar) é que se pode promover a interexportação de modelos terminológicos que não comprometam o domínio (postura interdisciplinar). O nível de consistência e de precisão da terminologia varia entre os diferentes campos e está relacionado com seu grau de maturidade, no sentido apresentado por Sager (1993).

A comunicação do conceito, tomando-se este elemento como unidade concentrada de conhecimento e como entidade epistemológica, depende de expressões linguísticas para que possa ocorrer (Dahlberg, 1978). A dependência do conceito de uma materialidade para fins de comunicação, implica em algum modo de delimitação ou de fixação do conceito num determinado signo. Impõe-se nesse caso um problema relativo à ambiguidade ou imprecisão da relação termo-conceito que deve ser abordado com os recursos teórico-metodológicos da terminologia.

Não se propõe e nem se espera que os conceitos possam ser terminologicamente estabilizados em princípios impraticáveis de univocidade de referência, conforme previa a terminologia clássica.

\section{Resultados e discussão}

A busca pelos termos "ontologia", "ontología" e "ontology", nos campos de título, resumos e palavras-chave das revistas que compõem o corpus documental resultou na recuperação total de dezoito artigos, doze identificados na Scire e seis na Ibersid.

Dentre os doze artigos localizados na Scire, três traziam o termo ontologia no título, nas palavras-chave e no resumo. Tais trabalhos são marcadamente conceituais. Em um artigo o termo apareceu no resumo e nas palavraschave e em sete deles, o termo ocorreu apenas no resumo.

Uma inconsistência na ferramenta de busca disponível na página da lbersid impediu a realização da busca nos mesmos moldes em que foi realizada na Scire para fins de comparação. A busca pelo termo "ontologia" nos campos de palavras-chave (términos de indexación) teve retorno zero. Dentre os seis artigos localizados, metade trazia o termo no título e no resumo.

Foi possível identificar ainda outras duas formas de inconsistência que requerem atenção editorial especial. Um artigo, publicado na Scire, com a presença do termo "ontologia" no campo de palavra-chave não foi recuperado pela ferramenta de busca, possivelmente por estar em forma adjetivada. O outro problema refere-se mais diretamente ao modo como o autor percebe e representa o próprio texto e ao modo como ele é incorporado ao periódico. Foram localizados, por meio de processos manuais, quatro artigos com frequência/ocorrência do termo superiores a 15 (16, 22, 36 e 41, respectivamente). Ou seja, esses artigos e mais outros 2 , com frequência/ocorrência menor, não são localizados pela ferramenta de busca porque seus autores não utilizaram o termo "ontologia" no título, no resumo ou em palavras-chave.

Em passo subsequente, cada ocorrência do termo foi investigada nos 18 artigos componentes do corpus de análise, para que pudessem ser devidamente categorizados. A possibilidade de tratamento do termo em mais de um aspecto no mesmo artigo fez com que alguns deles fossem enquadrados em mais de uma categoria. Trabalhou-se, conforme descrito no final da seção de introdução, com quatro categorias: a) conceitualização: discussões sobre o aparecimento do termo, sua etimologia e sua inserção na ciência da informação; b) comparação: cotejo de ontologias com outros instrumentos de orga- 
nização e representação do conhecimento; c) aplicação: exemplos e discussões sobre a utilização de ontologias, sem o caráter de ineditismo nelas; d) nova aplicação: apresentação e apreciação sobre a utilização de uma nova ontologia. A categorização pode ser visualizada no Quadro 1.

\begin{tabular}{|c|c|c|}
\hline Categoria & Periódico & Artigo \\
\hline \multirow{2}{*}{ Conceitualização } & Scire (3) & $\begin{array}{l}\text { Poli, 2002; Gnoli, } \\
\text { 2011; Moreira, } 2012\end{array}$ \\
\hline & Ibersid (1) & $\begin{array}{l}\text { Boccato; Ramalho; } \\
\text { Fujita, } 2008\end{array}$ \\
\hline \multirow{2}{*}{ Comparação } & Scire (4) & $\begin{array}{l}\text { Gilchrist, 2003; Lopez } \\
\text { Alonso, 1998; Lopez } \\
\text { Alonso, 2000; Moreira, } \\
2012\end{array}$ \\
\hline & Ibersid (2) & $\begin{array}{l}\text { Boccato; Ramalho; } \\
\text { Fujita, 2008; Pastor } \\
\text { Sánchez; Martínez } \\
\text { Méndez, } 2009\end{array}$ \\
\hline \multirow[b]{2}{*}{ Aplicação } & Scire (5) & $\begin{array}{l}\text { Lopez Alonso, 1998; } \\
\text { Poli, 2002; Delgado } \\
\text { Gómez, 2002; } \\
\text { García Marco, 2003; } \\
\text { Gnoli, } 2011\end{array}$ \\
\hline & Ibersid (3) & $\begin{array}{l}\text { Tennis; Calzada } \\
\text { Prado, 2007; Cosme; } \\
\text { Quaresma, 2007; } \\
\text { Pastor Sánchez; } \\
\text { Martínez Méndez, } \\
2009\end{array}$ \\
\hline \multirow[b]{2}{*}{ Nova aplicação } & Scire (1) & Casanovas, 2012 \\
\hline & Ibersid (3) & $\begin{array}{l}\text { Tennis; Calzada } \\
\text { Prado, 2007; } \\
\text { Rambaud, 2007; } \\
\text { Pastor Sánchez; } \\
\text { Martínez Méndez, } \\
2009\end{array}$ \\
\hline
\end{tabular}

Quadro 1. Categorização do termo "ontologia"

As questões conceituais e de comparação, que também são conceituais, pois buscam compreender o conceito de ontologia por meio de comparações com outros instrumentos de organização e representação do conhecimento como o tesauro e os sistemas de classificação, e.g., são encontradas principalmente na Scire $(n=7$, contra $\mathrm{n}=3$ na lbersid). Na grande categoria das aplicações, agrupando aplicação e nova aplicação, há empate na quantidade verificada, $6 \mathrm{em}$ cada periódico, com vantagem para a revista lbersid quando se tratam de novas aplicações.

Essa análise encontra eco nas propostas editorias dos dois periódicos. Considerando-se o escopo da Scire (representação e organização do conhecimento), esse periódico constitui-se numa consulta obrigatória para os que querem compreender o conceito de ontologia e suas aplicações.

A revista Ibersid, contudo, é orientada às questões mais gerais dos sistemas de informação e documentação. Tais questões podem até mesmo envolver, dependendo da forma de abordagem, questões teórico-conceituais sobre a organização e representação do conhecimento e seus instrumentos, mas, no que refere às ontologias, privilegiam as questões teórico-práticas.

\section{Considerações finais}

Defende-se, a partir da análise realizada, que o termo ontologia sofre um caso de metaterminologização, da filosofia para a ciência da informação (e da computação também) com manutenção do núcleo sêmico. No processo de metaterminologização, conforme exposto anteriormente, um determinado termo deixa uma terminologia na qual tem origem e passa a ser utilizado também numa outra terminologia. Isso pode ocorrer, segundo Barbosa (2005), de dois modos fundamentais: com ou sem manutenção do núcleo sêmico (traços de significado) comum aos termos das diferentes áreas.

Discussões sobre conceitos e sobre análise conceitual (Semidão, Almeida e Moreira, 2013) também requerem estudos sobre terminologia e sobre análise de domínio, pois os conceitos científicos são formados no interior de discursos científicos.

Quando surge um novo conceito, o primeiro desafio, no âmbito das línguas de especialidades, é encontrar uma expressão adequada para representá-lo, pois isso irá garantir ao conceito existência material. Invariavelmente, contudo, os conceitos nascem em contextos terminológicos imprecisos, pois nunca há tempo suficiente para que se espere por sua maturidade e pela identificação mais plena possível de suas características para denominá-lo, já que sem denominação não será possível operacionalizá-lo. Num segundo momento, cabe à terminologia e aos sistemas documentários, normalizar, de certo modo, o uso do termo, optando, por meio de análise, pela expressão mais adequada do conceito.

Em trabalhos posteriores, pretende-se aprofundar a análise com recurso de técnicas bibliométricas que permitam ampliar a análise da variáveis intervenientes na formação do conceito de ontologia na ciência da informação.

\section{Referências}

Barbosa, Maria Aparecida (2005). Terminologia e lexicologia: plurissignificação e tratamento transdisciplinar das 
unidades lexicais nos discursos etno-literários. // Revista de Letras. 1/2:27 (jan./dez.) 103-107.

Bardin, Laurence (2011). Análise de conteúdo. Lisboa: Edições 70, 2011.

Boccato, Vera Regina C.; Ramalho, Rogério Aparecido S.; Fujita, Mariângela S. L. (2008). A contribuição dos tesauros na construção de ontologias como instrumento de organização e recuperação da informação em ambientes digitais. // Ibersid. 2 (2008) 199-209. http://ibersid.eu/ojs/index.php/ibersid/article/view/2235/1 996 (2014-08-19)

Campos, Maria Luiza A (2004). Modelização de domínios de conhecimento: uma investigação de princípios fundamentais. // Ciência da Informação. 33:1 (jan./abr. 2004) 22-32. http://www.scielo.br/pdf/ci/v33n1/v33n1a03.pdf (2014-04-08)

Casanovas, Pompeu (2012). Algunas líneas de investigación en gestión del conocimiento jurídico: web semántica, ODR y derecho relacional. // Scire. 18:1 (ene./jun. 2012) 15-28. http://www.ibersid.eu/ojs/index. php/scire/article/view/3943/3672 (2014-08-20).

Cosme, Sandra; Quaresma, Paulo (2007). A contribution to improve the children's catalogue of the public libray. // Ibersid. 1 (2007) 323-330. http://ibersid.eu/ojs/index.php/ ibersid/article/view/3325/3086 (2014-08-20).

Delgado Gómez, Alejandro (2002). Organización de la información mediante el uso de lenguajes de modelado: viejos recursos para nuevas necesidades. // Scire. 8:1 (ene./jun. 2002) 55-86. http://www.ibersid.eu/ojs/index .php/scire/article/view/1159/1141 (2014-08-20).

García Marco, Francisco Javier (2003). La gestion del conocimiento: aplicacion a la promocion de la salud. // Scire. 9:1 (jul./dic. 2003) 151-170. http://www.ibersid.eu /ojs/index.php/scire/article/view/1463/1441 (2014-08-20).

Gilchrist, Alan (2003). Taxonomies and information architecture. // Scire. 9:1 (ene./jun. 2003) 37-46. http://www.iber sid.eu/ojs/index.php/scire/article/view/1453/1431 (201408-19)

Gnoli, Claudio (2011). Ontological foundations in knowledge organization: the theory of integrative levels applied in citation order // Scire. 17:1 (jan./jun. 2011) 29-34. http:// ibersid.eu/ojs/index.php/scire/article/view/4001 (201404-08)

Guarino, Nicola (1998). Formal ontology and information systems. // Guarino, Nicola (Ed.). Formal Ontology in information systems: proceedings of FOIS'98, Trento, ItaIy, 6-8 June 1998. Amsterdam: IOS Press, 1998. 3-15. http://migre.me/iHBjt (2014-04-08)

Hjorland, Birger (2008). What is knowledge organization (KO)? // Knowledge Organization. 35:2/3 (2008) 86-101.

Krieger, Maria da Graça; Finatto, Maria José B (2004). Introdução à terminologia: teoria e prática. São Paulo: Contexto, 2004

Lara, Marilda L. G (2009). Linguística documentária: seleção de conceitos. Tese (Livre-docência). Universidade de São Paulo, 2009

López Alonso, Miguel-Ángel (1998). El modelo conceptual en los sistemas de procesamiento de la información. //
Scire. 4:1 (ene./jun. 1998) 39-43. http://www.ibersid.eu/ ojs/index.php/scire/article/view/1085/1067 (2014-08-19).

López Alonso, Miguel-Ángel (2000). Las estructuras conceptuales de representación del conocimiento en internet. // Scire. 6:1 (ene./jun. 2000) 107-123. http://www.ibersid. eu/ojs/index.php/scire/article/view/1127/1109 (2014-0819).

Moreira, Walter (2012). Relações conceituais como ponto de inflexão entre linguagens documentais, terminologia e ontologias // Scire. 18:2 (jul./dic. 2012) 123-127. http:// www.ibersid.eu/ojs/index.php/scire/article/view/3946/371 3 (2014-08-19).

Pastor Sánchez, Juan Antonio; Martínez Méndez, Francisco Javier (2009). Aplicación de tesauros, taxonomías y ontologías en los sistemas de gestión de contenidos mediante tecnologías de la web semántica. // Ibersid. 3 (2009) 143-153. http://ibersid.eu/ojs/index.php/ibersid/ar ticle/view/3734/3495 (2014-08-20)

Poli, Roberto (2002). Glancing the problems of contemporary ontology // Scire. 8:1 (ene./jun. 2002) 17-40. http:// www.ibersid.eu/ojs/index.php/scire/article/view/1157/113 9 (2014-08-19).

Quine, Willard Van (1975). De um ponto de vista lógico. /l Os pensadores. São Paulo: Abril, 1975. 221-264.

Rambaud, Margarita G. (2007). A descriptrive algorithm for a wine tasting lexicon corpus. // Ibersid. 1 (2007) 313-321. http://ibersid.eu/ojs/index.php/ibersid/article/view/3324/3 085 (2014-08-20).

Sager, Juan Carlos (1993). Prólogo: la terminología: puente entre varios mundos. // Cabré, M. T. La terminología: teoria, metodologia, aplicaciones. Barcelona: Antártida/Empúries, 1993.

Semidão, Rafael Aparecido M.; Almeida, Carlos C.; Moreira, Walter (2013). Diretrizes para análise conceitual: as perspectivas de Hjorland, Dahlberg e Lakoff. // Ribeiro, Fernanda; Cerveira, Maria Elisa (Orgs.). Informação e/ou conhecimento: as duas faces de Jano: atas do I Congresso ISKO Espanha e Portugal e XI Congresso ISKO España. Porto - Portugal: Faculdade de Letras da Universidade do Porto - CETAC.MEDIA, 2013. 589-602. http://ocs.letras.up.pt/index.php/ISKO/ISKO/paper/view/1 47 (2014-04-08)

Smith, Barry (2003). Ontology. // Floridi, Luciano (Ed.). The blackwell guide to the philosophy of computing and information. Oxford: Blackwell, 2003. 155-166.

Staab, Steffen; Studer, Rudi. Preface. // Staab, Steffen; Studer, Rudi (Eds.). Handbook on ontologies. 2.ed. Berlin: Dordrecht, 2009. p. VII-XVI.

Tennis, Joseph T.; Calzada Prado, Javier (2007). Ontologies and the semantic web: problems and perspectives for LIS profesionals. // Ibersid. 1 (2007) 303-311. http://iber sid.eu/ojs/index.php/ibersid/article/view/3322/3083 (2014-08-20).

Enviado: 2014-04-09. Segunda versión: 2014-08-19 Aceptado: 2014-08-20. 